\title{
EL CONCEPTO DE BIENESTAR SOCIAL EN LA ECONOMÍA DEL BIENESTAR: UNA COMPLEJA RELACIÓN ENTRE ÉTICA, POLÍTICA Y ECONOMÍA*
}

\author{
THE CONCEPT OF SOCIAL WELFARE IN WELFARE \\ ECONOMICS: A COMPLEX RELATION BETWEEN \\ ETHICS, POLITICS AND ECONOMICS
}

\author{
Felipe Agustín Núñez Michea**
}

\begin{abstract}
Resumen
Este escrito tiene como objetivo global contribuir al análisis de los aspectos específicos en los que se dan las relaciones entre ética y economía. En particular, mostrar que la economía, considerada en su síntesis neoclásica, difícilmente puede sostener la distinción entre economía normativa y economía positiva, ya que toda noción de eficiencia ligada al bienestar social implica sostener proposiciones normativas en un sentido no trivial. No solo eso, los resultados más conocidos de la economía del bienestar, como el teorema de imposibilidad de Arrow, solo son válidos si se considera una estructura informacional de las funciones de utilidad muy restringida. En este sentido, es necesario utilizar un marco menos restrictivo para pensar y modelar distintos criterios de bienestar social. Considerando lo anterior, se muestra que la interacción entre economía y ética debe ser fortalecida, ya que el trabajo interdisciplinario permite evaluar políticas públicas desde perspectivas que no siempre están a la vista para las herramientas propias de los economistas.

Palabras clave: filosofía de la economía, economía del bienestar, bienestar social, ética, utilidad.
\end{abstract}

* ORCID: https://orcid.org/oooo-0oo3-357I-8I46. Agradezco al equipo del FONDECYT N. II8I4I4: "Análisis crítico del uso de modelos matemáticos en economía", cuyas discusiones y comentarios han sido de mucha utilidad para el desarrollo de este artículo.

** Universidad de Santiago. Santiago, Chile. E-Mail: fanunezmichea@gmail.com 


\begin{abstract}
The aim of this writing is to contribute to the analysis of the specific aspects in which the relationships between ethics and economics occur. In particular, to show that economics, considered in its neoclassical synthesis, can hardly sustain the distinction between normative economics and positive economics. Since any notion of efficiency linked to social welfare implies sustaining normative propositions in a non-trivial sense. Not only that, the most well-known results of welfare economics, such as Arrow's impossibility theorem, are only valid if a very restricted informational structure of utility functions is considered. In this sense, it is necessary to use a less restrictive framework to think and model differents criteria of social welfare. Considering this, it is shown that the interaction between economics and ethics must be strengthened, since interdisciplinary work allows evaluating public policies from perspectives that are not always visible to the economists' own tools.
\end{abstract}

Keywords: philosophy of economics, welfare economics, social welfare, ethics, utility.

Recibido: 2020-I2-29 Aceptado: 202I-O6-I5

\title{
I. Introducción
}

Las relaciones entre economía y ética son diversas y se dan en distintos niveles. Y es natural que así sea, después de todo, la economía es una ciencia social. Sin embargo, la mayoría de los economistas se sienten incómodos con esta relación. Es justamente esta incomodidad la que funda la distinción entre economía positiva y economía normativa, en la cual se deja en el lado de la economía positiva todos aquellos aspectos que conciernen a hechos económicos y al desarrollo de una ciencia económica, y dejando de lado de la economía normativa todos aquellos aspectos que conciernen al bienestar social, la distribución de la riqueza y la política económica.

En este escrito mostraré que la distinción entre economía normativa y economía positiva no puede sostenerse allí donde se hable de eficiencia como criterio de bienestar social. En este sentido, mostraré cómo se ha ido desarrollando el concepto de bienestar social en economía y qué perspectivas pueden abrirse considerando los supuestos teóricos clásicos. En particular, mostraré que la definición clásica de economía en términos del comportamiento de un agente racional ante medios escasos constriñe enormemente la posibilidad de incluso plantear los criterios de bienestar social que han sido clásicos en la tradición del pensamiento 
político. Así, concluyo que es necesario restablecer la relación entre ética y economía en tanto que sólo desde esta perspectiva es posible evaluar distintas políticas públicas y económicas que tienen consecuencias que a veces no son vistas por los modelos económicos en tanto la información que estos modelos contienen es acotada en un aspecto muy específico: las funciones de utilidad solo capturan información ordinal e intrapersonal.

Es importante decir que la distinción entre economía normativa y positiva, y en general las discusiones sobre metodología económica (Blaug 1985, Hausman 1992, Backhouse 2006, Reiss 2013) se dan sobre todo dentro del ámbito de la microeconomía, y en menor medida, en la macroeconomía (en parte por el microfundacionalismo (Hoover 20I0)), por lo que a esos aspectos me refiero fundamentalmente en este escrito, dejando abierta la posibilidad de que la econometría pueda ser un área de la economía que está libre de valor, más allá de las consideraciones respecto a las constricciones éticas del desarrollo de experimentos naturales y otros aspectos semejantes.

En lo que sigue mostraré, en la sección segunda, que la definición clásica de economía está profundamente ligada a la caracterización neoclásica de eficiencia y bienestar. En la sección tercera mostraré que el concepto de utilidad es equívoco y que la concepción económica del término, en tanto orden intrapersonal de preferencias, explica por qué es tan difícil incluso plantear los problemas respecto del bienestar social. En la sección cuarta mostraré cómo la concepción de utilidad en términos de preferencias lleva al teorema de imposibilidad de Arrow, pero que a pesar de eso, no es evidente qué debemos concluir de los resultados de este teorema. En la sección quinta mostraré cómo pueden plantearse criterios de bienestar social desde las herramientas económicas, aunque eso no sea suficiente para evitar la discusión filosófica. En la sección sexta mostraré otros caminos desarrollados desde la economía para contribuir al desarrollo de una economía del bienestar más potente. En la sección séptima mostraré cómo pueden relacionarse la economía con la ética para generar análisis más poderosos respecto de las políticas públicas y los límites del mercado por razones que el análisis económico simplemente no ve. Por último, en la sección octava, concluiré.

\section{La definición clásica de economía y el intento de una economía positiva}

A principios del siglo veinte la economía era un campo de batalla teórico respecto a los fines y los alcances del análisis económico. Consi- 
derando ese ambiente, y desde la batalla metodológica, surgió el texto An Essay on the Nature and Significance of Economic Science de Leonel Robbins (2007[1932]), que definió la economía como hoy la conocemos en su corriente principal o neoclásica: una ciencia que estudia el comportamiento humano como una relación entre los fines y los medios escasos que tienen usos alternativos. Hoy se podría agregar que la escasez es, también, respecto a la información.

Así definida, la economía parece ser una extensión, o quizás el mismísimo corazón, de una teoría general de la decisión. Después de todo, para generar un cálculo de decisión solo nos bastaría con encontrar una manera rigurosa de hablar sobre fines y de los medios para alcanzarlos. Así, respecto de un fin específico, por ejemplo, ganar el juego de lanzar un proyectil lo más lejos posible, y si se tienen fijos todos los factores excepto el ángulo de lanzamiento, entonces, existe un fin y un medio para lograr tal objetivo. El problema a solucionar es: ¿qué ángulo maximiza la distancia de lanzamiento? Una persona que tiene como fin ganar ese juego debe guiar su decisión de acuerdo con la solución al problema anteriormente especificado.

Si se agrega que los medios son escasos, sólo se restringe el problema diciendo que para los fines que se tienen, sean los que estos sean, los medios no alcanzan para lograrlos satisfactoriamente, por las razones que sean. Si, a su vez, los medios tienen usos alternativos, dejamos abierta la posibilidad a que puedan intercambiarse: este intercambio permite mejorar las condiciones iniciales en tanto los medios de un agente especificado sean más valiosos para otros y los medios de otros sean más valiosos para aquel.

El objetivo de este tipo de definición económica está en poner en el centro la idealización del agente racional que toma decisiones. El conjunto de estos agentes, en sus papeles de consumidores o empresarios, generan la mecánica de la teoría del consumidor y la mecánica de la teoría de la firma. A su vez, estas mecánicas generan la mecánica del equilibrio de mercado.

Así, la economía, considerando esta definición, se vuelve una teoría de la acción racional y, en ese sentido, es, por un lado, trivialmente normativa, pues norma lo que calificaría como acción racional de los agentes. Esto último puede leerse como un axioma, como una idealización útil o como un principio fundamental, de momento no es importante. Pero, por otro lado, no es una ciencia normativa en tanto que no norma lo que debe hacerse para aumentar el bienestar de los distintos agentes de la sociedad como conjunto. Según la definición clásica, no hay mane- 
ra de dar una medida a la importancia relativa del bien $\mathrm{x}$ para el agente i con la importancia del bien y para el agente $j$. Es decir, no es posible hacer comparaciones interpersonales de utilidad.

En este sentido, esta ciencia que estudia la relación entre medios y fines solo puede entregar un orden de preferencias intrapersonal, y este orden es representado por la idea de una función de utilidad ordinal para cada agente. En la siguiente sección explicaré algunos aspectos importantes sobre la idea de utilidad. De momento es importante tener presente que si se piensa desde órdenes de preferencias ordinales solo comparables intrapersonalmente, entonces lo que entendamos por eficiencia, término crucial para definir el concepto de equilibrio, solo puede considerar esta información para hacer economía propiamente científica, según la visión tradicional.

Sin duda, ha sido importante para los economistas determinar el aspecto científico de la economía, y la definición de Robbins permitiría establecer una diferencia clara entre lo que se ha llamado "economía positiva", la economía propiamente científica, que es una ciencia de la decisión en condiciones de escasez; y la economía normativa, hoy llamada, también, economía del bienestar, de carácter no científico. Un ejemplo claro de esta corriente y sus consecuencias se encuentra en las palabras del premio Nobel de economía, Robert Lucas: "Of the tendencies that are harmful to sound economics, the most seductive, and in my opinion the most poisonous, is to focus on questions of distribution." (Lucas 2003). Evidentemente, si es que la economía, definida à la Robbins no es normativa en un sentido interesante, es poco lo que pueden hacer los economistas respecto a preguntas sobre el bienestar y en general sobre el policy making. Como mostraré a lo largo de este trabajo, esta distinción entre economía positiva y normativa es imposible de sostener teóricamente, de hecho, de las suposiciones clásicas respecto de la teoría del consumidor y del equilibrio, sí surgen principios normativos que determinan una visión particular de lo que es el bienestar.

Justamente es importante, a este respecto, considerar en algo más de detalle el concepto clásico de eficiencia, la eficiencia de Pareto, que afirma que

Si X es un conjunto de estados sociales factibles, entonces el estado social factible $\mathrm{x}$ es óptimo en el sentido de Pareto si no admite mejoras paretianas. Es decir, si no existe otro estado social factible que le dé un nivel de bienestar mayor o igual a cada uno de los individuos, y estrictamente mayor para al menos uno. 
Esto es lo mismo que decir que un estado social factible es óptimo si es que nadie puede estar mejor sin dejar en peor situación a otro.

Como muestran Hausman y McPherson (p. 66), si aceptamos que I. no hay interdependencias en las funciones de utilidad de los agentes, es decir, que los agentes sólo se preocupan por la satisfacción de sus preferencias y que estas no dependen de las preferencias de los demás; 2. que hay un mercado para todos los bienes y servicios; 3 . no hay barreras de entrada ni salida en los mercados; $y$, 4. tanto compradores como vendedores son tomadores de precios, es decir, son incapaces de fijar los precios por sí mismos, entonces, nos encontramos en un equilibro perfectamente competitivo, que a su vez, es deseable en términos de eficiencia, ya que, como muestra el primer teorema del bienestar, todo equilibrio perfectamente competitivo es Pareto óptimo.

Por supuesto, esto no quiere decir que existan mercados perfectamente competitivos. Pero nos dice que, si la eficiencia es deseable, entonces es posible alcanzarla a través de los mercados perfectamente competitivos.

Ahora bien, la eficiencia de Pareto no es ni tan útil ni neutral para tomar decisiones económicas. En primer lugar, no es de utilidad para tomar decisiones respecto de situaciones que implican redistribución y que empeoran el bienestar de algunos para mejorar las condiciones de otros por razones de justicia. En el mismo sentido, es profundamente dependiente del statu quo, ya que si la situación inicial es injusta, no es mucho lo que puede ofrecer. En segundo lugar, no permite establecer una comparación entre los distintos óptimos de Pareto de un estado social dado.

Los alcances de la optimalidad de Pareto son estrechos en términos normativos, quizás por esa razón, la mayoría de los economistas lo consideran un principio de eficiencia mínimo que forma parte central de la teoría microeconómica y que no tendría que ver con aspectos normativos. Sin embargo, esta estrechez depende crucialmente de lo que se entiende por "utilidad" y cuál es su relación con la idea de bienestar.

\section{Sobre la idea de utilidad}

En el ámbito de la filosofía la idea de utilidad proviene de la literatura del utilitarismo inglés. Mucho se ha discutido respecto a qué se quiere decir con utilidad en esta corriente y, en general, en filosofía, pero no es fácil dar una respuesta unívoca. Además el concepto tiene usos distintos en filosofía y en economía, lo que ha provocado confusiones semánticas 
aún mayores, ya que estos usos se cruzan, generando problemas conceptuales que sólo dificultan la comprensión del alcance del concepto.

Esta clase de consideraciones permite que incluso hoy sea un tema abierto determinar qué se quiere decir con la palabra "utilidad" y, por sobre todo, determinar cuál es su relación con la idea de bienestar.

Para los economistas, en general, utilidad expresa bienestar en el sentido de que mientras más utilidad tengo, más bienestar tengo. Por supuesto, esta formulación es muy vaga, después de todo, ¿cómo cuantificar la cantidad de bienestar que me da determinado objeto frente a otro? ¿Este bienestar se traduce en placer o en alguna otra cosa? Por este tipo de consideraciones los economistas son reacios a utilizar comparaciones cardinales de utilidad, es decir, comparaciones que no solo ordenan la utilidad que otorga determinado bien de menor a mayor, sino que da una magnitud a esta, de manera que la diferencia entre estas magnitudes es información relevante: permite determinar cuánto más bienestar otorga un bien con respecto a otro. Incluso si es que utilizan este tipo de comparaciones, se asume que toda comparación puede hacerse en términos de dinero, asumiendo que esto es todo lo que importa en términos de bienestar.

Por esta razón, para evadir el uso de funciones cardinales de utilidad, los economistas utilizan funciones de utilidad como un mecanismo de representación de orden intrapersonal de preferencias. Esto quiere decir que dado que existen agentes con ciertas preferencias sobre cierto conjunto de objetos, y dado que sus preferencias son transitivas (es decir, cumplen con que si el bien a es al menos igualmente preferido $\mathrm{a} b$ $\mathrm{y}$ el bien $\mathrm{b}$ es al menos igualmente preferido a c, entonces es cierto que a es al menos igualmente preferido a c) y completas (es decir, el orden transitivo ocurre sobre todo el conjunto de objetos considerados), entonces es posible representar estas preferencias mediante una función de utilidad continua y creciente con el orden de preferencias. La diferencia de la utilidad que entregan dos bienes a un mismo sujeto no es información interpretable.

Por supuesto, menos aún es posible hacer una comparación entre la diferencia de utilidad que entrega el mismo bien para dos agentes distintos. ¿Sobre qué criterio se establecería esa comparación? Como he dicho, si la economía es por sobre todo una ciencia de la decisión, y si esta decisión es sobre preferencias, entonces, no hay ningún mecanismo que permita establecer una comparación de bienestar entre dos agentes distintos. Por esta razón, no se utilizan comparaciones interpersonales de utilidad, cardinales u ordinales, en economía. A excepción, por su- 
puesto, de la economía del bienestar, que como también ya he dicho, ha sido relegada fuera del terreno propiamente positivo o científico.

Así, al tratar las funciones de utilidad como mecanismo de representación de preferencias individuales, pueden evadirse muchas preguntas filosóficas en la práctica económica, pero solo es un desplazamiento del problema, después de todo, nada asegura que las preferencias de las personas sean lo que efectivamente deja en mejores condiciones a una persona según otro criterio de bienestar (Hausman 2018 trata este punto en profundidad). Esto muestra, como he dicho, que en economía solo es posible hablar de las preferencias de los agentes y que es imposible hacer comparaciones entre la cantidad de utilidad de un bien frente a otro, o cuánto se prefiere un bien a otro. Menos aún comparar la utilidad que le da determinado bien a dos agentes distintos. Por supuesto, con algunas suposiciones es posible hacer esas comparaciones, como aceptar que los agentes son egoístas y que todo lo que les importa es la cantidad de bienes que pueden obtener. Estas suposiciones no forman parte de los aspectos fundamentales del concepto de utilidad en economía, pero sí son fundamentales respecto a algunos modelos y teorías, como la teoría del consumidor.

Un punto respecto al final del párrafo anterior. La diferencia respecto a los modelos y las teorías en la economía es un asunto complicado que tiene muchas consecuencias, después de todo, determinar el núcleo de la economía neoclásica implica determinar lo que hay de teoría en ella y en ese sentido, comprender sobre qué se construyen los modelos. En escrito seguiré la distinción de Hausman (I992), según la cual una teoría es un conjunto de descripciones y explicaciones y predicciones que se expresan a través de conjuntos de proposiciones nomológicas. En este sentido, las teorías refieren al mundo a través de esas proposiciones, por lo que pueden ser verdaderas o falsas, y testeables. Los modelos, por otra parte, son más bien definiciones de sistemas y conceptos, por lo que son trivialmente verdaderos o falsos y, en cualquier caso, no testeables. No son afirmaciones, sino más bien suposiciones.

El papel de los modelos en economía, y esto no la distingue de las otras ciencias, es la exploración conceptual, el desarrollo de las aplicaciones teóricas, no la teoría en sí misma (Hausman I992, p. 80).

Pero el uso económico no es el único uso de la palabra "utilidad". De hecho, los utilitaristas ingleses se referían más bien a aquella propiedad que tenían ciertos objetos para producir placer, bienestar, bien, felicidad, etc. La utilidad no el bien en sí mismo, es una característica de ciertos objetos que producen bien, no el bien en sí mismo (Broome 2004). 
Este tipo de formulación deja abierta la pregunta sobre cuáles son aquellos objetos que producen placer, bienestar, felicidad, etc. Si simplemente aceptásemos que la pregunta es por aquellos objetos que producen bienestar, no avanzaríamos mucho, la palabra sigue siendo profundamente oscura. Como mostraré más adelante en este artículo, la filosofía política contemporánea ha hecho mucho para aclarar este debate. De momento es importante decir que la discusión sobre el bienestar se da en distintos niveles, después de todo, es muy distinto intentar dar una definición de bienestar individual a dar una definición de bienestar social. Si consideramos que bienestar es equivalente a satisfacción de preferencias, ¿es posible obtener algo así como una satisfacción social de preferencias?

Para comprender algunos aspectos de la noción de utilidad, es relevante volver a la discusión sobre economía positiva y normativa. Por supuesto, no todos los economistas están de acuerdo en esta distinción tajante, o, al menos, no están de acuerdo con que toda economía del bienestar sea una economía normativa. Este punto de vista expresa, por ejemplo, Hicks (1938) justamente tomando en cuenta la posición de Robbins. ¿Acaso es posible hablar de una economía del bienestar positiva? Esta es la perspectiva de la economía del bienestar paretiana y el criterio de Kaldor-Hicks.

$\mathrm{Si}$ es que no es posible establecer comparaciones interpersonales de utilidad y la información de las funciones de utilidad es sólo ordinal, entonces muchos caminos para la economía del bienestar han sido cerrados. Justamente por esta razón, la economía del bienestar paretiana asume las restricciones de la eficiencia de Pareto y la definición de economía que he considerado en este texto. En este sentido, se plantea un "cálculo utilitarista", como lo plantea Hicks (1938), la idea es que un estado social factible es preferible si quienes ganan en él pueden compensar a los que pierden. En realidad, este criterio es más exigente que el criterio de Pareto, ya que se juzga una decisión respecto del estado social independientemente de si las compensaciones se realizan o no. Pero tiene la fortaleza de que permite evaluar todo estado social según una mejora paretiana potencial, lo que extiende el terreno de la eficiencia paretiana. Ahora bien, evaluar estados sociales factibles es una cosa, y otra cosa, completamente distinta, es efectivamente recomendar un estado social independientemente de si se paga o no la compensación, ya que en este caso no se respetaría, de hecho, la eficiencia de Pareto y se haría un cálculo de costo-benificio agregado perfectamente utilitarista. Además ¿qué es lo que se está comparando en términos de utilidad? En 
general, se asume que estas comparaciones y compensaciones pueden hacerse en términos de dinero, lo que está lejos de no ser controvertido. Así, los límites de la eficiencia de Pareto siguen siendo los mismos y no solo eso, si se acepta completamente el criterio de Kaldor-Hicks, el criterio de elección de bienestar social coincide con un criterio utilitarista, lo que está, a todas luces, lejos de ser algo así como una economía del bienestar positiva.

¿Es acaso posible una economía del bienestar que se mantenga en los límites demarcados por Robbins? Y si no es posible, ¿qué camino le queda a la economía del bienestar? Esta pregunta intentaré responder en la siguiente sección.

\section{El concepto de función de utilidad social en economía y el teorema de imposibilidad de Arrow}

En I95I Kenneth Arrow publicó Social Choice and Individual Values (2012), iniciando la teoría de la elección social contemporánea y dándole una respuesta negativa a la primera pregunta con la que terminaba la sección anterior: no es posible una economía del bienestar que se mantenga en los límites demarcados por Robbins, o al menos no una útil. Dicho de otra manera, y más específicamente, Arrow demostró que no es posible generar una función de utilidad social que cumpla con ciertas características, a primera vista, razonables, a menos que esta función de utilidad social sea dictatorial. Ahora explicaré esto en lo esencial.

Arrow mostró, en su teorema de imposibilidad, que no existe función de bienestar social, es decir, una función que exprese las preferencias sociales, transitivas y completas, que cumpla los siguientes requisitos:

Condición de dominio irrestricto: el dominio de la función de bienestar social incluye todas las n-tuplas lógicamente posibles de los ordenamientos individuales sobre $\mathrm{x}$.

Condición de Pareto débil: para todo estado social x e y, si todos en la sociedad prefieren $\mathrm{x}$ a $\mathrm{y}$, entonces, $\mathrm{x}$ es socialmente preferido a $\mathrm{y}$.

Condición de independencia de las alternativas irrelevantes: si para dos perfiles de preferencias individuales, cada individuo tiene exactamente la misma preferencia con respecto a dos alternativas $\mathrm{x}, \mathrm{y}$, entonces las preferencias sociales con respecto a $\mathrm{x}$ e $\mathrm{y}$ deben ser las mismas para ambos perfiles.

Condición anti dictatorial: no ocurre que existe un agente en la sociedad tal que si ese agente prefiere $\mathrm{x}$ a $\mathrm{y}$, entonces $\mathrm{x}$ es preferido socialmente a $\mathrm{y}$. 
Puede pensarse en el teorema de imposibilidad como una generalización de la paradoja de Condorcet (una introducción a teorema de Arrow y la paradoja de Condorcet se encuentra en Peterson 2017), que muestra que las preferencias individuales de un conjunto de agentes, en conjunto con la aplicación de la regla de la mayoría como mecanismo de decisión, pueden generar preferencias sociales cíclicas, es decir, que no respetan transitividad, es decir, no se puede establecer un orden de preferencias agregadas.

Los requisitos de Arrow son poco exigentes en términos de la información necesaria sobre la medida y comparación de las preferencias de los agentes, de hecho, está en el terreno de Robbins. Quizás justamente por lo poco que exigen, la situación es problemática. Para enfrentar este problema es necesario sistematizar la estructura informacional que conllevan las preferencias, esto es algo que explicaré en la siguiente sección.

En realidad la demostración de Arrow está en términos axiomáticos, solo con órdenes de preferencia y sin utilizar funciones de utilidad. Sin embargo, ya que las funciones de utilidad solo son representaciones de preferencias, es posible generar pruebas con funciones de utilidad, y no solo eso, es posible leer la prueba desde la exigencia de la estructura informacional de las funciones.

Pero el problema de la estructura infomacional no es el único problema, cada uno de los supuestos es problemático de alguna manera, sobre todo, la condición de dominio irrestricto y la condición de alternativas irrelevantes. La condición de dominio irrestricto obliga a aceptar cualquier orden de preferencias individuales posibles, lo que es difícil de justificar en toda circunstancia. La condición de alternativas irrelevantes deja fuera toda función de utilidad social que sea sensible a ordenamientos de preferencias relacionales, lo que, sin duda, es bastante plausible.

Aceptar otro conjunto de supuestos, como acotar el conjunto de órdenes de preferencias individuales o negar la independencia de alternativas irrelevantes, es un camino para llegar a otros resultados, sin embargo, no se puede llevar muy lejos sin considerar más información en las funciones de utilidad. En cualquier caso, este no es el único problema: ¿el teorema de Arrow debe interpretarse como un resultado sobre la evaluación de estados sociales o como un resultado sobre la decisión de estados sociales? Si se considera que la búsqueda de una función de bienestar social es un mecanismo de evaluación de estados sociales, entonces aceptar la optimalidad de Pareto no es algo tan evidente. Además, ¿las preferencias deben leerse solo como preferencias o más bien como juicios, ya que si las personas votan, expresan sus preferencias en juicios? 
El debate sobre qué es lo que realmente afirma el teorema de imposibilidad no es trivial, después de todo, si es que leemos el teorema como un resultado sobre la evaluación de estados sociales sobre órdenes individuales de preferencias, entonces se debe concluir que el bienestar individual no puede ayudarnos para evaluar un estado socialmente mejor. Pero si el teorema es sobre la decisión de estados sociales, lo que nos dice es que no hay manera de conciliar las preferencias individuales. Si el teorema debe leerse como un mecanismo de decisión o evaluación sobre juicios, entonces las consecuencias también son distintas, y dependiendo de estas lecturas puede considerarse en más detalle la plausibilidad de los postulados de Arrow (para más detalles al respecto véase Hausman y McPherson 2006).

\section{Cómo salir del lecho de Procusto: la información de las funciones de utilidad}

Los requisitos de Arrow implican funciones ordinales de utilidad sin comparaciones interpersonales de ningún tipo. Desde un punto de vista filosófico, y quizás hasta semántico, es difícil imaginar que la noción de bienestar social, un concepto íntimamente ligado al concepto de justicia, sea pensable sin hacer comparaciones interpersonales de bienestar. Y si las funciones de utilidad expresan bienestar a través de las preferencias, entonces, parece ser que la economía simplemente se niega a pensar realmente en la idea de bienestar social.

Decir que las funciones de utilidad son ordinales y sin comparación interpersonal es decir que las preferencias personales solo se ordenan sin cuantificar la distancia de las preferencias. Además las preferencias no son comparables entre los agentes. Matemáticamente esto significa que a cada individuo se le puede asociar a una función de utilidad que cumpla con ser una transformación monótonamente creciente que preserve el orden de las preferencias. Tener esto en cuenta es importante, ya que determina la información que la sociedad usaría al tomar decisiones sociales.

Se pueden distinguir los grandes grupos de estructuras informacionales, en términos de la medida y la comparación del bienestar que llevan las funciones de utilidad, sistematizadas con unas pequeñas diferencias en Gaertner (2009), Roemer (1998):

AFC (Absolutely measurable and fully comparable): la única transformación posible es la función identidad. 
ONC (Ordinally measurable and noncomparable): todas las transformaciones posibles son monotonicamente crecientes, pero no necesariamente iguales para cada agente. De esta clase son las funciones de utilidad à la Arrow y las utilizadas en la economía en general.

CNC (Cardinally measurable and noncomparable): las transformaciones posibles son aquellas monotónicamente crecientes, entre ellas, las de la forma $a_{1} x+b_{1}, \ldots a_{n} x+b_{n}$. Donde $b_{i}$ es un número real y $a_{i}$ es un real positivo. Este grupo es especialmente importante porque es el grupo que permite las funciones de utilidad de Von Neumann Morgenstern, esenciales para modelar el utilitarismo de Harsanyi.

OFC (Ordinally full comparable): todas las transformaciones posibles son monotonamente crecientes, pero la misma para todo agente.

CUC (Cardinally measurable and unit comparable): las transformaciones son del tipo $a_{1} x+b, \ldots a_{n} x+b$. Este grupo permite medir diferencias de utilidades entre las personas, sin embargo no niveles de utilidad.

Por supuesto, la idea fundamental es que mientras más exigente es la estructura informacional, menos transformaciones hay disponibles. Una pregunta esencial aquí es si es suficiente la información que supone Arrow en la formulación del problema, ya que inevitablemente toda decisión social lleva en sí un criterio justicia. Por ejemplo, ¿es posible pensar en la justicia sin un concepto de bienestar claro y comparable entre los agentes?, ¿podemos pensar en un criterio de bienestar solamente ordinal, o implica cardinalidad?, ¿cuánta es la información mínima que deben llevar las funciones de utilidad para pensar en la justicia?

Estas preguntas no tienen una respuesta clara, pero lo que sí es cierto, es que la información que capturan las funciones de utilidad en el esquema de Arrow no es suficiente para modelar ni pensar las nociones de bienestar social clásicas en filosofía. Como ejemplo puede considerarse el utilitarismo y el igualitarismo de Rawls.

John Harsanyi propuso una forma de modelar el utilitarismo a través de una perspectiva axiomática. Sin duda, el término "utilitarismo" en Harsanyi es peculiar y ha llevado a dudas semánticas (Greaves 20I7), otros (Roemer 2008) han insistido en que no es propiamente una posición utilitarista. Y hay razones para plantear estas críticas. Después de todo, el utilitarismo de Harsanyi es bastante específico, utiliza una estructura de funciones de utilidad cardinales comparables mediante diferencias en el marco de las funciones de utilidad de Von Neumann Morgenstern, es decir, se utilizan preferencias sobre loterías (al respecto véase Petersen 20I7). Sin embargo, puede llamarse utilitarismo en un sentido muy general, ya que busca maximizar la suma de las utilidades de los agentes. 
En realidad, la posición de Harsanyi se puede dividir en dos partes, ya que ofrece dos argumentos diferentes para defender una posición utilitarista. La primera es especialmente interesante por su conexión directa con la posición rawlsiana. El segundo argumento es matemáticamente más técnico y no me detendré en él, para los propósitos de este texto las consecuencias no difieren.

Harsanyi asume que los agentes son racionales en el sentido de Von Neumann Morgenstern, así, sus funciones de utilidad tienen las características propias de las decisiones bajo incertidumbre. Es necesario considerar que los agentes están en una situación de incertidumbre porque Harsanyi piensa el utilitarismo como la decisión que toma un observador imparcial de la sociedad: un observador que pondera cada utilidad individual y cada posición social de manera idéntica. En este sentido, el observador imparcial es un agente que no sabe ni quién es ni cuál es su posición social. En este aspecto la propuesta de Rawls se parece mucho a la de Harsanyi. Dicho de otra manera, como los agentes no saben en qué posición están, los jugadores actúan como un observador imparcial y le otorgan igual probabilidad a cada posición por el principio de razón insuficiente. Así, la evaluación de un estado social $\mathrm{x}$ cualquiera, asumiendo que el peso de los agentes es idéntico, es igual a la suma de las utilidades individuales dividido por el número de agentes. Y como la utilidad de los agentes depende de su posición social, esta es una utilidad esperada. Así, el utilitarismo maximiza la suma de las utilidades esperadas de los agentes, dividido por el total de ellos.

En cualquier caso, esto no ha estado exento de críticas, ya que si bien es cierto que todo utilitarista asume el igual trato de los individuos, el proyecto de Harsanyi implicaba la derivación de una teoría ética desde principios matemáticos. Sin embargo, Harsanyi no da un fundamento formal desde el cual derivar este igual trato, más bien lo presupone, $\mathrm{y}$ al hacerlo, debe dar un fundamento para las comparaciones interpersonales de utilidad, ya que de otra forma es imposible pensar cómo el observador imparcial evalúa (Weymark 199I).

Pensar la filosofía rawlsiana desde la perspectiva de la teoría de la elección social también nos obliga a usar comparaciones interpersonales de utilidad. La descripción completa de los resultados de la posición original, según Rawls, no es de importancia para los propósitos de este texto, pero importa el hecho de que para modelar el principio de diferencia rawlsiano, aspecto fundamental de la filosofía política de Rawls, (las desigualdades solo son permitidas si es que estas mejoran el bien- 
estar de aquellos que están en peores condiciones) es necesario utilizar aquellas comparaciones.

En economía este tipo de consideraciones, como las del principio de diferencia, se pueden modelar a través de la regla de decisión maximin:

Maximin: en una sociedad tal que todos los agentes están indiferentes entre el estado social $\mathrm{x}$ y el estado social $\mathrm{y}$, a excepción de los agentes i y j, $x$ es estrictamente socialmente preferido a $y$ si $u_{i}(y)<u_{i}(x)<u_{j}(x)<u_{j}(y)$.

El problema del enfoque axiomático ralwsiano es que describe una posición moral igualitarista. Sin embargo, deja de lado el hecho de que Ralws intenta establecer un concepto procedimental de justicia, es decir, que no dependa de las posiciones morales. El problema con la aproximación axiomática es que no soluciona el problema de por qué nuestro criterio de justicia debería satisfacer tales o cuales axiomas, que es justamente la cuestión central. Por lo mismo, un enfoque axiomático que se haga cargo del problema de la justicia procedimental debe considerar que los agentes de la sociedad tienen distintas sensibilidades morales, no los policy makers, es decir, los distintos individuos siguen distintas normas que se expresan a través de axiomas, al momento de decidir lo justo.

Distintas descripciones axiomáticas se han mostrado (Roemer I998; Gaertner 2009; Fleurbaey and Maniquet 20II) y mostrar los axiomas y teoremas que se deducen de esos caminos implica más de lo que puedo permitirme aquí, sin embargo, lo importante es que estos enfoques no solucionan el problema de por qué un criterio de justicia socialmente determinado debería seguir tales axiomas, o lo que es lo mismo, de por qué la sociedad en su conjunto debería aceptar un conjunto de axiomas, que es justamente la cuestión central. En definitiva, se asume que de alguna manera las posiciones igualitaristas como la de Rawls, o las utilitaristas como la de Harsanyi, están en igualdad de condiciones en tanto pueden describirse matemáticamente. Sin embargo, se deja a criterio de los policy makers la tarea de pensar en cuál es la correcta, pero si ese el caso, aún no se soluciona el problema de establecer los fundamentos, en términos formales, de un criterio de justicia.

Me parece que la crítica anterior es suficientemente fuerte como para mostrar que no es suficiente el análisis económico: el análisis ético debe considerarse en distintos niveles, en primer lugar, para mostrar qué es lo que realmente quieren decir las proposiciones en economía del bienestar, como el teorema de imposibilidad de Arrow, y para evaluar proposiciones éticas donde el análisis económico de la teoría de la elección social simplemente no alcanza. 


\section{Los límites de la teoría de la elección social}

Como mostré en la sección anterior, el problema fundamental de la teoría de la elección social, para el análisis con espíritu filosófico, es que no permite evaluar el conjunto de axiomas a utilizar como criterio de bienestar, quedando a decisión del policy maker la elección de estos sin mayores consideraciones. Sin embargo, ya que la idea de bienestar es una noción normativa, parece que justamente esto es lo que debe discutirse en primer lugar, este aspecto surge desde un problema económico, por lo que no puede simplemente eludirse. Dejar este aspecto como una cuestión subjetiva (Jehle y Reny 20II, p. 290) es un límite que sólo puede plantearse el economista que no tiene las herramientas para evaluar estos aspectos, o que simplemente quiere negarse a aceptar que la economía en estos aspectos es esencialmente normativa.

Este tipo de consideraciones no son aplicables sólo a la teoría de la elección social, el corazón de la economía del bienestar. Los mismos problemas surgen con el enfoque de teoría de juegos cooperativa, que tiene la ventaja de expresar los problemas de decisión en términos de un contrato, con un punto de desacuerdo, que es expresado por un vector de utilidades dado, en el que los agentes no llegan a un acuerdo, y un conjunto de puntos que expresan mejoras a ese status quo. El punto de solución al problema planteado recibe el nombre de "punto de solución al problema de la negociación", por lo que parece expresar razonablemente la perspectiva hobbesiana del contrato social como mutuo beneficio.

Existen soluciones clásicas al problema de la negociación, como la solución de Nash, la solución de Kalai-Smorodinsky y la solución de Kalai, sin embargo, el problema con este tipo de formulaciones coincide con lo visto en la perspectiva de teoría de la elección social: el punto de solución al problema es determinado por una función que lo expresa, sin embargo, nuevamente, no hay manera de evaluar las distintas soluciones entre sí. Tal vez para un economista esta exigencia parecerá demasiado, sin embargo, es una pregunta legítima, e ineludible, para el tipo de problemas en los que se ve enfrentado el economista que se dedica a la economía del bienestar.

Otras miradas han sido propuestas desde la economía para repensar en la idea de bienestar. La teoría de juegos evolutiva ha mostrado su utilidad para describir la justicia en términos de coordinación, y pueden ser útiles para mostrar cómo generar cambios sociales en términos de equilibrios evolutivamente estables. 
Desde esta perspectiva son especialmente interesantes, para filósofos, los trabajos de Skryms (2014) y Binmore (2005). Sin embargo, este tipo de consideraciones más bien reduccionistas, pues el concepto de justicia, por ejemplo, se reduce a una solución evolutiva de los problemas de coordinación, no logran explicar ni evaluar el hecho de que en una sociedad pluralista hay distintas miradas sobre el bienestar. No es suficiente con explicar las condiciones actuales, pese a que puede ser útil para resolver cuestiones relacionadas con la aplicación de políticas públicas. En cualquier caso, hay cierto grado de ingenuidad en las intenciones profundamente reduccionistas respecto de los problemas éticos (véase, por ejemplo, Güth y Hartmut 20I0; Calvin y O'Connor 20I9).

Consideraciones parecidas pueden hacerse con respecto a los avances de la economía conductual. Hoy puede decirse con bastante certeza que el uso del homo economicus como una idealización útil para los modelos económicos no es tan útil después de todo: los agentes tienen preferencias que la mayoría de las veces expresan una preocupación por los demás (Lisciandra 2018) y no solo eso, en muchas circunstancias las preferencias tienen características morales (Yaari y Bar-Hillel 1984). Sin duda esto permite limitar el poder explicativo de los modelos económicos, utilizados a veces sin más como verdades de la ciencia económica. Sin embargo, esto no quiere decir que los economistas estén profundamente equivocados al considerar que los agentes son maximizadores de utilidad o que tienen preferencias transitivas. Me parece que esto sigue siendo una idealización útil, solo basta con considerar, como se hace ampliamente hoy, que las funciones de utilidad pueden expresar preocupaciones por los demás y que los agentes no son necesariamente perfectamente egoístas, y por lo tanto, la noción de eficiencia en términos de Pareto, es mucho más compleja de lo que parece.

Lo que quisiera dejar en claro con estas reflexiones es que la economía enfrenta constantemente problemas éticos, por lo que la filosofía política y la ética no es irrelevante para el estudio de los economistas ni menos para la evaluación de las políticas públicas. Sobre este punto profundizaré en la siguiente sección.

\section{Perspectivas en el cruce de filosofía y economía}

La unión entre filosofía y economía es íntima, solo que con vericuetos que generan relaciones complejas e indirectas. Aun así, es razonable pensar que no se pueden comprender los problemas económicos sin una perspectiva filosófica. Es imposible comprender los aspectos funda- 
mentales de La riqueza de las naciones sin comprender las ideas de Smith en La teoría de los sentimientos morales. De la misma manera, no es posible comprender la dinámica del Capital de Marx sin entender su trasfondo sistemático de corte hegeliano, o comprender la distancia entre la economía marxista frente a la economía neoclásica sin analizar los presupuestos de lo que se entiende por valor, ni comprender la perspectiva austriaca sin entrar en el debate sobre cómo debe entenderse la idea de libertad. En general, estos son debates conceptuales y en todos los casos anteriores, profundamente filosóficos. Solo el intento constante de la economía por transformarse en una ciencia fundamentalmente matemática (una historia al respecto se encuentra en Weintraub 2002) ha podido pretender lo contrario.

En este escrito no he entrado en la discusión respecto de cómo las economías heterodoxas han criticado la visión neoclásica a través de sus distintas visiones sobre qué es el valor o de una idea particular de bienestar. Sin duda, esta es una manera de criticar la economía de la corriente principal. Sin embargo, ese camino no permite necesariamente comprender de manera analítica los problemas teóricos de la economía neoclásica, por el contrario, solo nos permite buscar maneras de evitar sus consecuencias a un nivel general. Por esta razón he preferido mostrar que incluso dentro de los límites de la economía neoclásica no es posible hacer economía positiva, al menos nada que integre el concepto de eficiencia y el concepto de bienestar social. Así, no es posible evitar los debates que hacen de la economía, justamente, una ciencia social.

Esta no es una perspectiva nueva, los alcances de la ética y la filosofía política, y su utilidad en el análisis económico y las políticas públicas, han sido mostrados en extenso por Hausman y McPherson (2006). En lo fundamental, Hausman y McPherson concluyen que la filosofía puede ayudar en la evaluación de políticas públicas allí donde el análisis económico no alcanza, o incluso allí donde el análisis económico asume ciertas ideas de bienestar que pasan por neutrales. La mayoría del tiempo las preguntas normativas se confunden con aspectos puramente subjetivos, como si ante este tipo de cuestiones no se pudiese establecer una evaluación a través de una discusión racional y, por lo tanto, una resolución. Esto simplemente no es cierto. El análisis conceptual es de utilidad justamente en estos aspectos y, en este sentido, la filosofía es útil no sólo para el análisis de los aspectos económicos de la vida, los mercados y las relaciones sociales que en ellos se instancian, sino que también para el análisis de la teoría económica como tal. Sin duda, los economistas pueden utilizar con provecho las herramientas conceptuales que la 
filosofía ha desarrollado, de la misma forma que los filósofos han utilizado, con enormes frutos, las herramientas conceptuales de los economistas. En este sentido, he mostrador que el concepto de bienestar que se maneja en economía es problemático por al menos dos razones. En primer lugar, surge de un análisis teórico muy particular, pero profundamente arraigado: el análisis de las preferencias individuales a través de funciones de utilidad ordinales e incomparables interpersonalmente; y en segundo lugar, el formalismo matemático implicado (que da pie al teorema de imposibilidad de Arrow) no es suficiente para determinar las conclusiones que pueden extraerse del teorema de imposibilidad y por ende de la idea de bienestar.

En términos filosóficos esto implica que deben analizarse en detalle los supuestos formales, las relaciones y las consecuencias de otros criterios de bienestar clásicos en la filosofía política. E incluso más allá: ¿es posible ir más allá del relativismo en el que se estanca el análisis de los diferentes criterios de bienestar, es posible generar un modelo formal para expresar este problema? Me parece que pensar la posición original rawlsiana considerando las doctrinas comprensivas razonables (Rawls 1996) es un buen punto de partida para pensar el problema.

A niveles más amplios, lo anterior implica que las políticas públicas deben tomar en cuenta los aspectos éticos de las decisiones sociales. Después de todo, la elección de políticas públicas es un problema, por sobre todo, político, y, por ende, ético. La reflexión respecto a la regulación, o incluso prohibición, de ciertos mercados ha sido un tema profundamente discutido por filósofos contemporáneos como Debra Satz (20IO) y Michael Sandel (2012), y comprender los límites morales del mercado no es una tarea intuitiva. Requiere el análisis conceptual que permite explicar y evaluar lo que está en juego en la esfera económica y cómo esta esfera se relaciona con todos los aspectos de la vida, en particular, con nuestra visión de la democracia y de los derechos humanos.

\section{Conclusión}

He mostrado por qué la distinción entre economía normativa y economía positiva es insostenible en un dominio importante de la teoría económica. En particular, no puede sostenerse allí donde se utiliza alguna noción de eficiencia, como en los teoremas fundamentales del bienestar o en los problemas distributivos.

A partir de lo anterior se hace explícito que la definición clásica de economía en términos de agentes racionales con medios escasos no per- 
mite generar una economía del bienestar atractiva para resolver los problemas propios de este dominio: problemas de decisiones de políticas públicas y de criterios de bienestar social. A través del análisis del teorema de imposibilidad de Arrow se mostró que es posible describir criterios de bienestar con las herramientas económicas, pero esto implica dejar de lado las constricciones que se establecen sobre las funciones de utilidad según la corriente principal. Sin embargo, pese a que esto es útil para describir criterios de bienestar social, los problemas de evaluación de ellos no tienen solución desde esta perspectiva. Es aquí donde la filosofía mantiene una discusión fecunda y útil para la discusión pública.

\section{Referencias bibliográficas}

Arrow, Kenneth J. (20I2). Social Choice and Individual Values. Vol. I2. Yale university press.

Backhouse, Roger E. (2006). Explorations in Economic Methodology: From Lakatos to Empirical Philosophy of Science. Routledge.

Blaug, Mark (1985). "La metodología de la economía o cómo explican los economistas, Madrid.” Alianza.

Binmore, Ken (2005). Natural Justice. Oxford university press.

Broome, John (2004). Ethics out of Economics. Cambridge University Press.

Cochran, Calvin, and O'Connor, Cailin (2019). "Inequality and inequity in the emergence of conventions." Politics, Philosophy \& Economics I8.3: 264-28I. DOI:_https://doi.org/Io.II77/I470594XI982837I

Fleurbaey, Marc, and Maniquet, François (201I). A Theory of Fairness and Social Welfare. Vol. 48. Cambridge University Press.

Gaertner, Wulf (2009). A primer in Social Choice Theory: Revised Edition. Oxford University Press.

Greaves, Hilary (20I7). "A reconsideration of the Harsanyi-Sen-Weymark debate on utilitarianism."Utilitas 29.2: I75-213. DOI: https://doi. org/IO.IOI7/So95382081600oI69

Güth, Werner, and Kliemt, Hartmut (2010). "What ethics can learn from experimental economics - If anything." European Journal of Political Economy 26.3: 302-310.

Hausman, Daniel M. (1992). The Inexact and Separate Science of Economics. Cambridge University Press.

Hausman, Daniel, and McPherson, Michael (2006). Economic Analysis, Moral Philosophy, and Public Policy. Cambridge University Press. 
Hausman, Daniel M. (2018). "The Bond between Positive and Normative Economics." Revue d'economie politique I28.2: 19I-208. DOI: https:// doi.org/I0.39I7/redp.282.0I9I

Hicks, John R. (1939). “The Foundations of Welfare Economics.” The Economic Journal 49.196: 696-712. DOI: I0.2307/2225023

Hoover, Kevin D. (2010). "Idealizing reduction: The microfoundations of macroeconomics." Erkenntnis 73.3: 329-347. DOI: I0.I007/sI0670-0IO-9235-I

Jehle, G. A., and Reny, P. J. (20II). "Advanced Microeconomic Theory (Third)." Essex: Pearson Education Limited.

Lisciandra, Chiara (2018). "The role of psychology in behavioral economics: The case of social preferences." Studies in History and Philosophy of Science Part A 72: II-2I. DOI: https://doi.org/IO.IOI6/j.shpsa.20I8.0I.0IO

Lucas, Robert E. (2004). "The industrial revolution: Past and future." Annual Report Essay. Federal Reserve Bank of Minneapolis. Recuperado: https://www.minneapolisfed.org/article/2004/the-industrial-revolution-past-and-future

Peterson, Martin (2017). An Introduction to Decision Theory. Cambridge University Press.

Rawls, John (1996). Liberalismo político. FCE .

Reiss, Julian (2013). Philosophy of Economics: A Contemporary Introduction. Routledge.

Robbins, Lionel (2007). An Essay on the Nature and Significance of Economic Science. Ludwig von Mises Institute.

Roemer, John E. (1998). Theories of Distributive Justice. Harvard University Press.

Roemer, John (2008). "Harsanyi’s Impartial Observer Is Not a Utilitarian" contained in Fleurbaey, Marc, Maurice Salles, and John Weymark (edited)." Justice, Political Liberalism, and Utilitarianism-Themes from Harsanyi and Rawls .

Sandel, Michael J. (2012). What Money Can't Buy: The Moral Limits of Markets. Macmillan.

Satz, Debra (2010). Why Some Things Should Not Be for Sale: The Moral Limits of Markets. Oxford University Press.

Skyrms, Brian (20I4). Evolution of The Social Contract. Cambridge University Press.

Weintraub, E. Roy (2002). How Economics Became a Mathematical Science. Duke University Press. 
Weymark, John A. (199I). "A reconsideration of the Harsanyi-Sen debate on utilitarianism." In Jon Elster \& John E. Roemer (eds.), Interpersonal Comparisons of Well-Being. Cambridge University Press. pp. 255.

Yaari, Menahem E., and Bar-Hillel, Maya (1984). "On Dividing Justly." Social Choice and Welfare I.I: I-24. DOI: https://doi.org/IO.IOO7/ BFoo297056 